\title{
New Opportunities with Oval-shaped Silicon Drift Detectors for High-Throughput EDX Analysis in Electron Microscopy
}

\author{
A. Niculae ${ }^{1}$, A. Bechteler ${ }^{1}$, R. Eckhardt ${ }^{1}$, K. Hermenau ${ }^{1}$, A. Liebel ${ }^{1}$, G. Lutz ${ }^{2}$, A. Schöning ${ }^{1}$, H. Soltau ${ }^{1}$, \\ L. Strüder ${ }^{2}$ \\ 1. PNDetector GmbH, Otto-Hahn-Ring 7, München, Germany \\ 2. PNSensor GmbH, Otto-Hahn-Ring 7, München, Germany
}

The progress made over the years in electron microscopy has pushed the instrument abilities towards new frontiers. Higher beam intensities, smaller spot sizes, fast and accurate detectors - all these features enable sample imaging and elemental analysis at atomic scale resolution in real time. Often equipped with one or more Energy Dispersive X-Ray (EDX) detectors, the analytical capabilities of the electron microscopes have gained more and more significance over the last years.

For high resolution electron microscopes like TEMs or high resolution SEMs, the distance between the pole piece and the sample plane (so-called working distance) is often very small; thus when using an EDX detector of a given size, the maximum attainable solid angle for collecting the $\mathrm{x}$-ray signal is limited by the minimum distance at which the detector can approach the sample point. For these cases, using an oval-shaped detector of reduced size in the vertical dimension enables a closer positioning of the detector with respect to the sample point (see Figure 1a) and therefore a significant increase of the solid angle. The solid angle can be further increased by using a multi-detector configuration. Figure $2 \mathrm{~b}$ shows a dual EDX detector system with two oval-shaped detectors arranged at $180^{\circ}$ around the polepiece.

At PNDetector we have been working since many years on the optimization of the detector geometry with respect to the maximum achievable solid angle. One of the first detectors especially designed for high solid angle application is the annual multi-channel detector configuration with central hole called Rococo2. This detector configuration has been presented several times in the past [1] and is not the subject of this contribution. More recently, several single-channel detector configurations of oval shape have been developed for high solid angle EDX analysis in TEM and SEM. Figure 2 shows three such configurations of the so-called SDD Oval-Line with active areas of $60 \mathrm{~mm}^{2}, 100 \mathrm{~mm}^{2}$ and $200 \mathrm{~mm}^{2}$.

The $100 \mathrm{~mm}^{2}$ oval shaped detector is already in use for more than five years. Depending on the poleshoe configuration, solid angle values of up to $1 \mathrm{sr}$ can be achieved. Combining the detector with the $\mathrm{SDD}^{\text {plus }}$-FET technology [2], energy resolution values down to $125 \mathrm{eV}$ at $5.9 \mathrm{keV} \mathrm{x}$-ray energy are measured (see plot in Figure 3). Using the detector in a windowless configuration, the sensitivity for light elements greatly improves, enabling light element detection down to Si-L lines $(90 \mathrm{eV})$.

In this contribution we will presents new results obtained with the various oval-shaped detector configurations. The advantages (large solid angle, excellent optimum energy resolution) and the limitations (e.g. ballistic deficit due to the large aspect ratio) of these detectors will be discussed.

References:

[1] A. Liebel et al., paper presented at M\&M 2013 in Indianapolis

[2] A. Niculae et al., paper presented at M\&M 2013 in Indianapolis. 


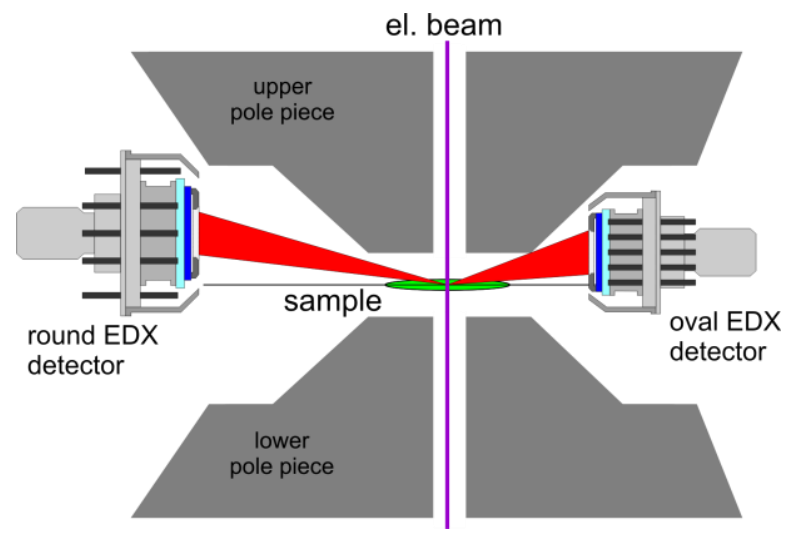

(a)

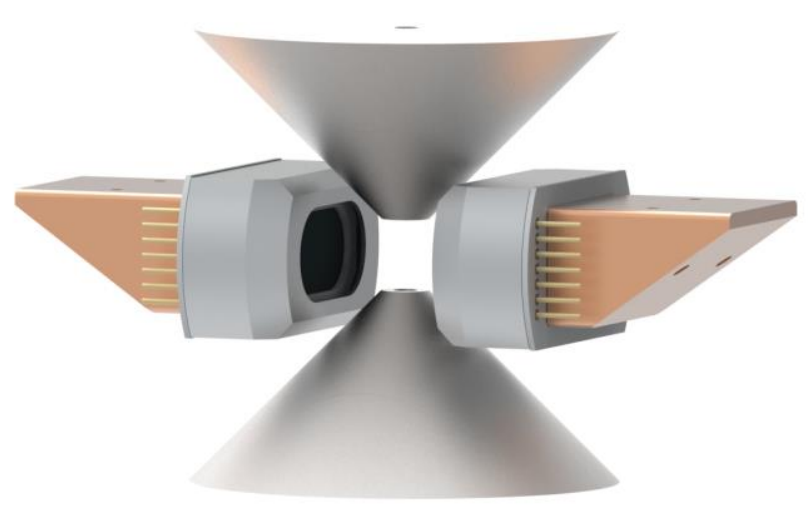

(b)

Figure 1. (a) Schematic view of a pole-piece with a round EDX detector on the left and an oval-shaped detector of similar area on the right hand side; (b) a dual EDX detector system arranged at $180^{\circ}$ around the microscope pole-piece.
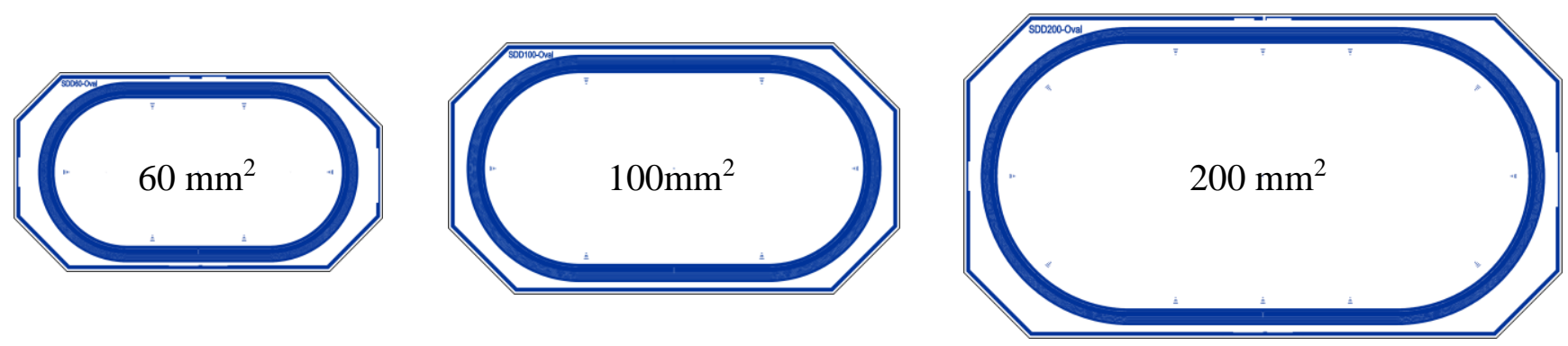

Figure 2. Three oval-shaped SDD geometries with total active areas of 60,100 and $200 \mathrm{~mm}^{2}$.
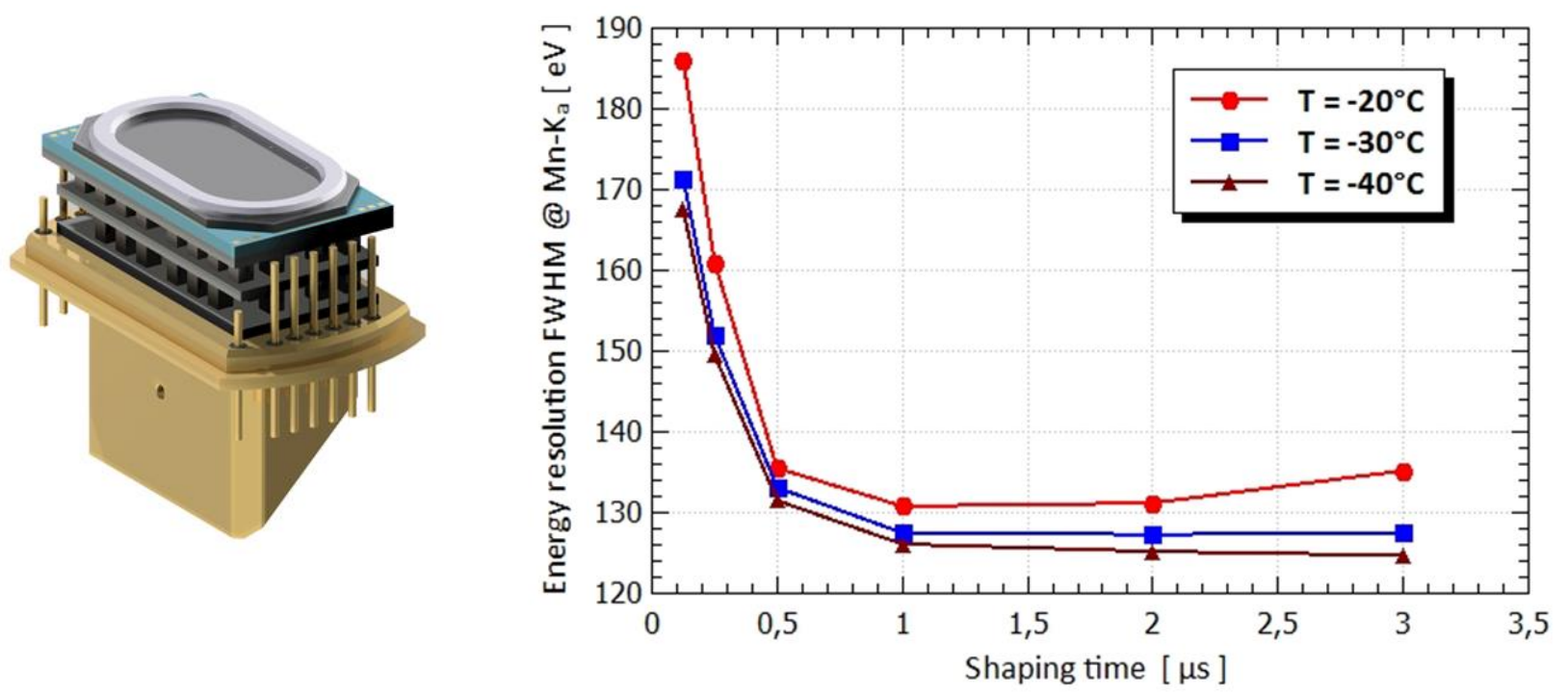

Figure 3. Spectroscopic performance of an $100 \mathrm{~mm}^{2}$ oval-shaped $\mathrm{SDD}^{\text {plus }}$ detector versus shaping time at different sensor temperatures. 